\title{
Observation of and model for nonlinear mode conversion in a non-neutral plasma
}

\author{
Grant W. Hart \\ grantwhart@gmail.com \\ Bryan G. Peterson \\ bryan_peterson@byu.edu \\ Ross L. Spencer \\ ross_spencer@byu.edu
}

Follow this and additional works at: https://scholarsarchive.byu.edu/facpub

Part of the Astrophysics and Astronomy Commons, and the Physics Commons

\section{Original Publication Citation}

Hart, Grant W., Ross L. Spencer, and Bryan G. Peterson."Observation of and model for nonlinear mode conversion in a non-neutral plasma." Physics of Plasmas 1 (23): 2738-2747.

\section{BYU ScholarsArchive Citation}

Hart, Grant W.; Peterson, Bryan G.; and Spencer, Ross L., "Observation of and model for nonlinear mode conversion in a non-neutral plasma" (2003). Faculty Publications. 486.

https://scholarsarchive.byu.edu/facpub/486

This Peer-Reviewed Article is brought to you for free and open access by BYU ScholarsArchive. It has been accepted for inclusion in Faculty Publications by an authorized administrator of BYU ScholarsArchive. For more information, please contact ellen_amatangelo@byu.edu. 


\title{
Observation of and model for nonlinear mode conversion in a non-neutral plasma
}

\author{
Grant W. Hart, Ross L. Spencer, and Bryan G. Peterson \\ Department of Physics and Astronomy, Brigham Young University, Provo, Utah 84602
}

(Received 24 February 2003; accepted 17 April 2003)

\begin{abstract}
The nonlinear interaction of the two lowest Trivelpiece-Gould modes in a non-neutral plasma has been observed. Because of coupling in the nonlinear terms of the continuity and momentum equations, the two modes can exchange energy and convert one to the other. This can be modeled using the cold fluid equations and the averaging method. Experimentally, this process always stops with the lower frequency mode dominating the final state. Numerical integration of the model suggests that this occurs because the higher frequency mode is more strongly damped than the lower frequency mode. (C) 2003 American Institute of Physics. [DOI: 10.1063/1.1581882]
\end{abstract}

\section{INTRODUCTION}

Like most finite-sized systems, a non-neutral plasma in a Malmberg-Penning trap has a spectrum of normal modes of oscillation. For a long, thin plasma, the lowest frequency electrostatic Trivelpiece-Gould (TG) oscillations are almost harmonically related in frequency and wavelength, ${ }^{1,2}$ making it possible to easily couple them to each other.

In this paper we designate the mode numbers by the index $j$ where $j$ is the number of half-wavelengths of the mode that fit into the plasma in the $z$-direction. The two modes of interest in this paper are the two lowest modes, the $j=1$ mode (with frequency and wavenumber $\omega_{1}$ and $k_{1}$ ), where the whole plasma sloshes back and forth between the two ends, and the $j=2$ mode (with $\omega_{2}$ and $k_{2}$ ), where the two ends of the plasma oscillate in opposite directions, making a breathing-type of oscillation.

Experimentally, whenever a large amplitude $j=2$ mode is created in the plasma, within a short time (of the order of a hundred cycles) it converts into a $j=1$ oscillation. This paper is intended to explain this phenomenon.

In this paper we first discuss the physical mechanism of the coupling and then a more complete theory which predicts how these modes should couple with each other. We then present experimental measurements of this effect and compare them with the predictions of the theory.

\section{THEORY}

\section{A. Illustration of the coupling mechanism}

This effect can be calculated using cold fluid theory as the basic model. The equations used are the continuity equation, momentum equation and Poisson's equation:

$$
\begin{aligned}
& \frac{\partial n}{\partial t}=-\nabla \cdot(n \mathbf{v}), \\
& n m \frac{\partial \mathbf{v}}{\partial t}+\mathbf{v} \cdot \nabla \mathbf{v}=-q n \nabla \Phi,
\end{aligned}
$$

$$
\nabla^{2} \Phi=-\frac{q n}{\epsilon_{0}}
$$

where $q$ is the charge on the particles ( $-e$ for electrons).

The mechanism for the coupling between these modes is the beating between them as they interact in the nonlinear $\nabla \cdot(n \mathbf{v})$ term in the continuity equation and the $\mathbf{v} \cdot \nabla \mathbf{v}$ term in the momentum equation. These terms contain the products of the spatial and temporal sinusoidal dependencies of these modes, which can be rewritten in terms of the sum and difference frequencies. When those sum or difference terms match the spatial and temporal dependence of a given mode, then they can cause that mode to grow or decay, depending on the relative phase of those terms and the mode.

To illustrate roughly how this coupling works using only the continuity equation, let us examine the nonlinear term in that equation. The starting point is the assumed expressions for the density and velocity of the standing waves in the plasma. Each mode is assumed to have a frequency and wavenumber given by $\omega_{j}$ and $k_{j}$ and density and velocity $n_{j}$ and $v_{j}$. We also assume for simplicity that these are the only two modes that are of importance in this plasma. The perturbed density for these modes can be expressed as

$$
\begin{aligned}
& n_{1}=\overline{n_{1}} \sin \left(\omega_{1} t+\phi_{1}\right) \sin \left(k_{1} z\right), \\
& n_{2}=\overline{n_{2}} \sin \left(\omega_{2} t+\phi_{2}\right) \cos \left(k_{2} z\right) .
\end{aligned}
$$

The quantity $\overline{n_{j}}$ represents the slowly varying (relative to the mode frequency) density amplitude of the mode. Similarly, the fluid velocity of each mode can be written as

$$
\begin{aligned}
& v_{1}=V_{1} \cos \left(\omega_{1} t+\phi_{1}\right) \cos \left(k_{1} z\right), \\
& v_{2}=-V_{2} \cos \left(\omega_{2} t+\phi_{2}\right) \sin \left(k_{2} z\right) .
\end{aligned}
$$

The coefficients $\overline{n_{j}}$ and $V_{j}$ are connected by the linear continuity equation,

$$
\frac{\partial n_{j}}{\partial t}=-\frac{\partial}{\partial z}\left(n_{0} v_{j}\right)
$$


where the velocity in the equilibrium state is assumed to be zero. Applying this gives

$$
V_{j}=\frac{\overline{n_{j}}}{n_{0}} \frac{\omega_{j}}{k_{j}},
$$

which can be substituted into Eqs. (6) and (7) above to give

$$
\begin{aligned}
& v_{1}=\frac{\overline{n_{1}}}{n_{0}} \frac{\omega_{1}}{k_{1}} \cos \left(\omega_{1} t+\phi_{1}\right) \cos \left(k_{1} z\right), \\
& v_{2}=-\frac{\overline{n_{2}}}{n_{0}} \frac{\omega_{2}}{k_{2}} \cos \left(\omega_{2} t+\phi_{2}\right) \sin \left(k_{2} z\right) .
\end{aligned}
$$

Taking the total density $n=n_{0}+n_{1}+n_{2}$ and the total velocity $v=v_{1}+v_{2}$ and putting them into the continuity equation produces a number of terms:

$$
\begin{aligned}
\frac{\partial n_{1}}{\partial t}+ & \frac{\partial n_{2}}{\partial t}+\frac{\partial}{\partial z}\left(n_{0} v_{1}+n_{0} v_{2}+n_{1} v_{1}+n_{2} v_{1}+n_{1} v_{2}\right. \\
& \left.+n_{2} v_{1}\right)=0 .
\end{aligned}
$$

Calculating $\partial n_{1} / \partial t$ produces two terms:

$$
\begin{aligned}
\frac{\partial n_{1}}{\partial t}= & \overline{n_{1}} \omega_{1} \cos \left(\omega_{1} t+\phi_{1}\right) \sin \left(k_{1} z\right) \\
& +\frac{\partial \overline{n_{1}}}{\partial t} \sin \left(\omega_{1} t+\phi_{1}\right) \sin \left(k_{1} z\right) .
\end{aligned}
$$

The first term on the right-hand side of Eq. (12) cancels with the $n_{0} v_{1}$ term from the $\partial / \partial z(n v)$ part of the continuity equation. Similarly, the equivalent part of $\partial n_{2} / \partial t$ cancels with the $n_{0} v_{2}$ part.

The forms of $n$ and $v$ from Eqs. (4)-(5) and (9)-(10) above are inserted into these equations and the trigonometric sum and difference formulas are used to rewrite the products. We now make use of the fact that for a long plasma $\omega_{2}$ $\approx 2 \omega_{1}$ and $k_{2} \approx 2 k_{1}$, so $\omega_{2}-\omega_{1} \approx \omega_{1}$ and $k_{2}-k_{1} \approx k_{1}$. We also group all the terms that have the time and spatial dependence of $n_{1}$ and $n_{2}$ together, since those are the only terms that can give a slow, long term change in the mode amplitudes. This gives

$$
\begin{aligned}
\left(\frac{\partial \overline{n_{1}}}{\partial t}-\frac{\overline{n_{1}} \bar{n}_{2}}{2 n_{0}} \omega_{1} \cos \Phi\right) \sin \left(\omega_{1} t+\phi_{1}\right) \sin \left(k_{1} z\right)+\left(\frac{\partial \overline{n_{2}}}{\partial t}\right. \\
\left.+\frac{\overline{n_{1}^{2}}}{2 n_{0}} \omega_{1} \cos \Phi\right) \sin \left(2 \omega_{1} t+\phi_{2}\right) \sin \left(2 k_{1} z\right)+\cdots=0,
\end{aligned}
$$

where $\Phi=2 \phi_{1}-\phi_{2}$, which can be thought of as the relative phase between the two modes. The $\cdots$ term represents the terms with spatial and temporal dependence that do not match that of either $\overline{n_{1}}$ or $\overline{n_{2}}$.

This equation implies that

$$
\begin{aligned}
& \frac{\partial \overline{n_{1}}}{\partial t}=\frac{\overline{n_{1}} \overline{n_{2}}}{2 n_{0}} \omega_{1} \cos (\Phi), \\
& \frac{\partial \overline{n_{2}}}{\partial t}=-\frac{\overline{n_{1}^{2}}}{2 n_{0}} \omega_{1} \cos (\Phi) .
\end{aligned}
$$

Note that when $\Phi$ is between $\pi / 2$ and $-\pi / 2$, the $j=1$ mode will grow at the expense of the $j=2$ mode, and the reverse will occur when $\Phi$ is outside of that range. The maximum growth rate for $\overline{n_{1}}$ occurs when $\Phi=0$.

Similar relationships to those above can be derived for $\partial \overline{n_{3}} / \partial t$ and $\partial \overline{n_{4}} / \partial t$. We will not have a need for them in this paper because the frequency mismatch between the relevant modes is relatively large in the experiment. This produces small oscillations in the amplitudes of the modes, but no wholesale conversion of one mode to another.

\section{B. A more complete analysis}

In the previous illustration, the phase was treated as an independent parameter that was not dynamically moved by the equations. If the momentum equation is included in the analysis it can model the evolution of the phase as well as the amplitudes. We will also include damping for both modes.

We have included linear damping in our analysis because we observe such damping experimentally for small amplitude modes. These modes are large enough that Landau damping is almost certainly nonlinear, but we feel that the observed damping is mostly caused by external resistance, particularly in the amplifiers that supply the confinement voltages. Since the TG modes are positive energy modes, resistive energy dissipation will cause them to damp. Particle in cell simulations support this interpretation of the damping.

The continuity equation, the momentum equation and the Poisson equation cannot just be solved in one dimension, since for long, thin plasmas like ours the radial part of the Poisson equation dominates over the axial part. Since the exact dispersion relation is not important, we will not attempt to derive it. We will just note that since we are discussing Trivelpiece-Gould normal modes of the plasma, the Poisson equation will take the form

$$
\left(-k_{r}^{2}-k^{2}\right) \phi=-\frac{n q}{\epsilon_{0}},
$$

and the dispersion relation obtained by linearizing Eqs. (1)(3) is then

$$
\omega^{2}=\frac{\omega_{p}^{2}}{k_{r}^{2}+k^{2}} k^{2},
$$

where $k_{r}$ is the eigenvalue associated with the the radial direction and $k$ is the usual $k$ for sinusoidal $z$ dependence. This allows the term for the potential in the momentum equation to be replaced with a density term. The set of equations becomes

$$
\begin{aligned}
& \frac{\partial n}{\partial t}=-\frac{\partial}{\partial z}(n v), \\
& \frac{\partial v}{\partial t}+v \frac{\partial v}{\partial z}=-\frac{\omega_{p}^{2}}{\left(k_{r}^{2}+k^{2}\right)} \frac{\partial n}{\partial z}=-\frac{\omega^{2}}{k^{2}} \frac{\partial n}{\partial z}-\gamma v .
\end{aligned}
$$


We have used Eq. (16) in the last step in Eq. (18). For simplicity of notation, our density variable in Eqs. (17)-(18) and in the remainder of the paper is actually the dimensionless variable $n / n_{0}$.

We again assume that the normalized density and velocity can be written in the form

$$
\begin{aligned}
& n=1+n_{1}(t) \sin \left(k_{1} z\right)+n_{2}(t) \cos \left(k_{2} z\right), \\
& v=v_{1}(t) \cos \left(k_{1} z\right)+v_{2}(t) \sin \left(k_{2} z\right) .
\end{aligned}
$$

Note that all of the time dependence is in the coefficients. These forms are put into Eqs. (17) and (18), the product terms are converted into their sum and difference frequencies, and all terms with the same spatial dependence are gathered together. This gives four equations for the time dependence:

$$
\begin{aligned}
& \dot{n}_{1}=k_{1} v_{1}+\frac{k_{1}}{2} n_{1} v_{2}+\frac{k_{1}}{2} n_{2} v_{1}, \\
& \dot{n}_{2}=-k_{2} v_{2}-\frac{k_{2}}{2} n_{1} v_{1}, \\
& \dot{v}_{1}=-\frac{\omega_{1}^{2}}{k_{1}} n_{1}+\left(\frac{k_{1}-k_{2}}{2}\right) v_{1} v_{2}-\gamma_{1} v_{1}, \\
& \dot{v}_{2}=\frac{\omega_{2}^{2}}{k_{2}} n_{2}+\frac{k_{1}}{2} v_{1}^{2}-\gamma_{2} v_{2} .
\end{aligned}
$$

In these equations we have introduced the mode-dependent damping coefficients $\gamma_{1}$ and $\gamma_{2}$. These terms model the experimentally observed damping of the modes.

These equations include the entire time dependence of the oscillations, but we are really only interested in the slow (compared to the oscillation frequency) variation of the amplitude and the relative phase of the two modes. In order to extract this information, we use the averaging method on these equations. ${ }^{3}$

We apply this method by converting these four firstorder equations into two second-order equations for $v_{1}$ and $v_{2}$. This is done by taking the time derivatives of Eqs. (23) and (24). Equations (21) and (22) for $\dot{n}_{1}$ and $\dot{n}_{2}$ are then substituted into the resulting equations. We then solve Eqs. (23) and (24) for $n_{1}$ and $n_{2}$ and use them to eliminate all references to $n$. The resulting pair of equations is

$$
\begin{aligned}
\ddot{v}_{1}+\omega_{1}^{2} v_{1}= & \left(k_{1}-\frac{k_{2}}{2}\right) \dot{v}_{1} v_{2}+\left(\frac{k_{1}-k_{2}}{2}-\frac{k_{2}}{2} \frac{\omega_{1}^{2}}{\omega_{2}^{2}}\right) v_{1} \dot{v}_{2} \\
& -\frac{k_{1}\left(k_{1}-k_{2}\right)}{4} v_{1} v_{2}^{2}+\frac{k_{1} k_{2}}{4} \frac{\omega_{1}^{2}}{\omega_{2}^{2}} v_{1}^{3} \\
& -\left(\frac{k_{2}}{2} \frac{\omega_{1}^{2}}{\omega_{2}^{2}} \gamma_{2}-\frac{k_{1}}{2} \gamma_{1}\right) v_{1} v_{2}-\gamma_{1} \dot{v}_{1},
\end{aligned}
$$

$$
\begin{aligned}
\ddot{v}_{2}+\omega_{2}^{2} v_{2}= & k_{1}\left(\frac{\omega_{2}^{2}}{2 \omega_{1}^{2}}+1\right) v_{1} \dot{v}_{1}-\frac{k_{1}\left(k_{1}-k_{2}\right)}{4} \frac{\omega_{2}^{2}}{\omega_{1}^{2}} v_{2} v_{1}^{2} \\
& -\frac{k_{1}}{2} \frac{\omega_{2}^{2}}{\omega_{1}^{2}} \gamma_{1} v_{1}^{2}-\gamma_{2} \dot{v}_{2} .
\end{aligned}
$$

We now assume that the quantities $v_{1}$ and $v_{2}$ can be written as

$$
\begin{aligned}
& v_{1}=a_{1} \cos \left(\omega_{1} t+\phi_{1}\right), \\
& v_{2}=-a_{2} \cos \left(\omega_{2} t+\phi_{2}\right),
\end{aligned}
$$

where $a_{j}$ is the time-dependent amplitude of mode $j$ and $\phi_{j}$ is that mode's time-dependent phase. Note that a nonzero $\dot{\phi}_{j}$ represents a frequency shift for mode $j$. We have chosen to include the negative sign on the expression for $v_{2}$ in order to simplify the interpretation of the relative phase of our data, which is related to $n_{j}$ and not $v_{j}$. This follows the time dependence given in Eqs. (4)-(7).

If we call the right-hand side (rhs) of Eq. (25) $F$ and the rhs of Eq. (26) $G$, then the averaging method in the form of Ref. 3 tells us that

$$
\begin{aligned}
& \dot{a}_{1}=-\frac{1}{\omega_{1}}\left\langle\sin \left(\omega_{1} t+\phi_{1}\right) F\right\rangle, \\
& \dot{a}_{2}=-\frac{1}{\omega_{2}}\left\langle-\sin \left(\omega_{2} t+\phi_{2}\right) G\right\rangle \\
& \dot{\phi}_{1}=-\frac{1}{\omega_{1}}\left\langle\frac{\cos \left(\omega_{1} t+\phi_{1}\right) F}{a_{1}}\right\rangle+\omega_{1}, \\
& \dot{\phi}_{2}=-\frac{1}{\omega_{2}}\left\langle\frac{-\cos \left(\omega_{2} t+\phi_{2}\right) G}{a_{2}}\right\rangle+\omega_{2},
\end{aligned}
$$

where the angle brackets indicate a time average over one period of the quantity inside of them, i.e.,

$$
\langle X\rangle=\frac{1}{2 \pi / \omega} \int_{t}^{t+2 \pi / \omega} X d t^{\prime} .
$$

Performing the indicated integrals yields the following results for $a_{1}$ and $a_{2}$ :

$$
\begin{aligned}
\dot{a}_{1}= & \frac{3}{8} k_{1} a_{1} a_{2} \cos (\Phi)-\frac{k_{1}}{16 \omega_{1}}\left(2 \gamma_{1}-\gamma_{2}\right) a_{1} a_{2} \sin (\Phi) \\
& -\frac{1}{2} \gamma_{1} a_{1}, \\
\dot{a}_{2}= & -\frac{3}{8} k_{1} a_{1}^{2} \cos (\Phi)-\frac{k_{1}}{4 \omega_{1}} \gamma_{1} a_{1}^{2} \sin (\Phi)-\frac{1}{2} \gamma_{2} a_{2} .
\end{aligned}
$$

Note that these equations depend only on the relative phase $\Phi=2 \phi_{1}-\phi_{2}$, and not on $\phi_{1}$ or $\phi_{2}$ separately. Therefore Eqs. (31) and (32) can be combined to produce a single equation for $\Phi$. This gives 


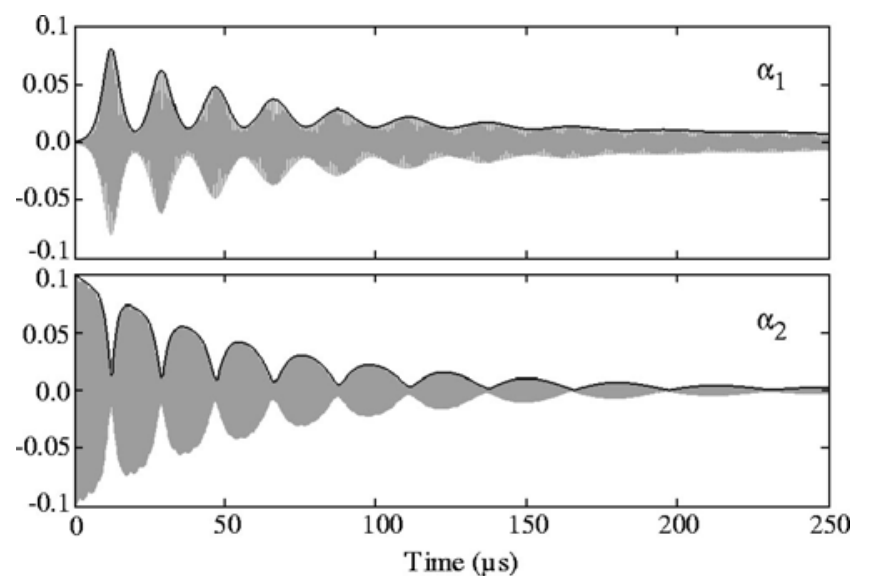

FIG. 1. A comparison of the solution of the full set of equations and the averaged equations. The gray lines indicate the solution of the full set of equations, including the high frequency time dependence. The solid black line is the amplitude calculated from the averaged equations. Clearly the amplitude of the oscillation is closely matched to the amplitude calculated from the averaged equations.

$$
\begin{aligned}
\dot{\Phi}= & -\frac{k_{1}}{4 \omega_{1}}\left(\gamma_{1} \frac{a_{1}^{2}}{a_{2}}+\frac{1}{2} a_{2}\left(2 \gamma_{1}-\gamma 2\right)\right) \cos (\Phi) \\
& +\frac{3}{8} k_{1}\left(\frac{a_{1}^{2}}{a_{2}}-2 a_{2}\right) \sin (\Phi)-\frac{k_{1}^{2}}{32 \omega_{1}}\left(a_{1}^{2}-4 a_{2}^{2}\right)+\delta \omega,
\end{aligned}
$$

where $\delta \omega=2 \omega_{1}-\omega_{2}$ is the degree of mismatch between the linear frequencies of the two modes.

It is convenient to express these equations in terms of the dimensionless variables $\alpha_{j}=v_{j} / v_{p}$ where $v_{p}=\omega_{1} / k_{1}$ is the phase velocity of the lowest mode. If we examine the connection between the density and velocity variables given in Eq. (8), we see that our dimensionless velocity is equal to the dimensionless density for each mode, i.e., $n_{j}=\alpha_{j}$. Expressing these three equations in terms of $\alpha_{1}, \alpha_{2}$ and $\Phi$ we find

$$
\begin{aligned}
\dot{\alpha}_{1}= & \frac{3}{8} \omega_{1} \alpha_{1} \alpha_{2} \cos (\Phi)-\frac{1}{16} \alpha_{1} \alpha_{2}\left(2 \gamma_{1}-\gamma_{2}\right) \sin (\Phi) \\
& -\frac{1}{2} \alpha_{1} \gamma_{1}, \\
\dot{\alpha}_{2}= & -\frac{3}{8} \omega_{1} \alpha_{1}^{2} \cos (\Phi)-\frac{1}{4} \gamma_{1} \alpha_{1}^{2} \sin (\Phi)-\frac{1}{2} \gamma_{2} \alpha_{2}, \\
\dot{\Phi}= & \frac{3}{8} \omega_{1}\left(\frac{\alpha_{1}^{2}}{\alpha_{2}}-2 \alpha_{2}\right) \sin (\Phi)-\frac{1}{4}\left(\gamma_{1} \frac{\alpha_{1}^{2}}{\alpha_{2}}+\frac{1}{2} \alpha_{2}\left(2 \gamma_{1}\right.\right. \\
& \left.\left.-\gamma_{2}\right)\right) \cos (\Phi)-\frac{\omega_{1}}{32}\left(\alpha_{1}^{2}-4 \alpha_{2}^{2}\right)+\delta \omega .
\end{aligned}
$$

Figure 1 shows how the results of integrating the averaged Eqs. (36)-(38) compared with the results of integrating the exact equations given in Eqs. (21)-(24). The amplitude curve calculated from the averaged set closely follows the envelope of the oscillations calculated from the exact set. When the amplitude becomes large enough that the ampli- tude changes significantly during one oscillation, then the assumptions of the averaging method are violated and the two sets of equations do not agree well. This starts to occur at an initial value of $\alpha_{2}$ of about 0.2 .

\section{Consequences of the model}

As we will see in Sec. III, the experimental data show that this system often phase-locks, i.e., the relative phase between the two modes approaches some particular value and stays there. Experimentally, the final state also has the $j=1$ mode much larger than the $j=2$ mode as they both decay away. We now explore this behavior analytically using the averaged equations.

Several of the terms in the averaged equations are negligible compared to the others, so the first step is to eliminate them from the equations. The ratio of the second term to the first in Eq. (36) is

$$
\frac{\left(2 \gamma_{1}-\gamma_{2}\right)}{6 \omega_{1}} \tan (\Phi) \text {. }
$$

Since these modes are typically only weakly damped, this is a small number unless $\Phi$ is close to $\pm \pi / 2$. We will ignore this term now and show later that this is justified. By similar reasoning, the second term in Eq. (37) can be ignored. The ratio of the second term in Eq. (38) to the first term is of order $\left(\gamma_{1} / \omega_{1}\right) \cot (\Phi)$, so it is small unless $\Phi$ is near 0 or $\pi$. The ratio of the third term to the first in Eq. (38) is of order $\alpha_{2} / \sin (\Phi)$, which is also a small number unless $\Phi$ is near 0 or $\pi$.

The averaged equations with only the significant terms are

$$
\begin{aligned}
& \dot{\alpha}_{1}=\frac{3}{8} \omega_{1} \alpha_{1} \alpha_{2} \cos (\Phi)-\frac{1}{2} \gamma_{1} \alpha_{1}, \\
& \dot{\alpha}_{2}=-\frac{3}{8} \omega_{1} \alpha_{1}^{2} \cos (\Phi)-\frac{1}{2} \gamma_{2} \alpha_{2}, \\
& \dot{\Phi}=\frac{3}{8} \omega_{1}\left(\alpha_{1}^{2} / \alpha_{2}-2 \alpha_{2}\right) \sin (\Phi)+\delta \omega .
\end{aligned}
$$

We start with the assumption that $\gamma_{2}>2 \gamma_{1}$, in line with the experimentally observed data. Under these conditions numerical experimentation with Eqs. (40) to (42) shows that, not surprisingly, $\alpha_{2}$ decreases faster than $\alpha_{1}$. This means that late in time the first term in Eq. (40) becomes negligible compared to the second term and Eq. (40) reduces to

$$
\dot{\alpha}_{1}=-\frac{1}{2} \gamma_{1} \alpha_{1}
$$

with the solution

$$
\alpha_{1}=\overline{\alpha_{1}} e^{-\gamma_{1} t / 2},
$$

where $\overline{\alpha_{1}}$ is the amplitude of mode 1 at the time where this approximation becomes valid.

At this late time we can use the solution given in Eq. (44) in Eq. (41), which becomes 


$$
\dot{\alpha}_{2}=-\frac{3}{8} \omega_{1} \bar{\alpha}_{1}^{2} e^{-\gamma_{1} t} \cos (\Phi)-\gamma_{2} \alpha_{2}
$$

Then Eq. (42) becomes

$$
\dot{\Phi}=\frac{3}{8} \omega_{1} \frac{\overline{\alpha_{1}^{2}} e^{-\gamma_{1} t}}{\alpha_{2}} \sin (\Phi)+\delta \omega .
$$

Equations (44)-(46) have a phase-locked state (defined as $\dot{\Phi}=0$ ) where

$$
\tan \left(\Phi_{0}\right)=\frac{-2 \delta \omega}{-\gamma_{e}}
$$

and

$$
\alpha_{20}=-\frac{\frac{3}{4} \omega_{1} \overline{\alpha_{1}^{2}} e^{-\gamma_{1} t} \cos \left(\Phi_{0}\right)}{\gamma_{e}},
$$

where the subscript 0 indicates the phase-locked state and $\gamma_{e}$ is defined to be

$$
\gamma_{e}=\gamma_{2}-2 \gamma_{1}
$$

The minus signs are used in Eq. (47) to emphasize the fact that this phase is in the third quadrant, where both the sine and cosine are negative.

Using standard perturbation techniques one can show that this phase-locked state is stable and that the system approaches that state as

$$
e^{-\gamma_{2} t / 4 \pm i\left(\sqrt{16 \delta \omega^{2}+\gamma_{e}^{2}} / 4\right) t} .
$$

This state also has the property that the quantity $\alpha_{1}^{2} / \alpha_{2}$ is a constant.

For future reference the expressions for $\sin (\Phi), \cos (\Phi)$ and $\alpha_{1}^{2} / \alpha_{2}$ are

$$
\begin{aligned}
& \sin (\Phi)=-\frac{2 \delta \omega}{\sqrt{4 \delta \omega^{2}+\gamma_{e}^{2}}}, \\
& \cos (\Phi)=-\frac{\gamma_{e}}{\sqrt{4 \delta \omega^{2}+\gamma_{e}^{2}}}, \\
& \frac{\alpha_{1}^{2}}{\alpha_{2}}=\frac{4}{3} \sqrt{4\left(\frac{\delta \omega}{\omega_{1}}\right)^{2}+\left(\frac{\gamma_{e}}{\omega_{1}}\right)^{2}} .
\end{aligned}
$$

We can now verify dropping the second terms from Eqs. (36) and (37). If the form of $\sin (\Phi)$ from Eq. (51) is put into Eq. (39), the result is of order $\delta \omega / \omega_{1}$, and therefore can be dropped. There could be problems with the terms we dropped from Eq. (38) if $\delta \omega \ll \gamma_{e}$, but that is not the case for our experimental parameters. Typically $\delta \omega$ is about ten times a large as $\gamma_{e}$.

We note here that the time it takes to achieve a phaselocked state depends on the initial values of $\alpha_{1}$ and $\alpha_{2}$. For our typical case of small $\alpha_{1}$ and larger $\alpha_{2}$, the smaller $\alpha_{2}$ is, the longer it takes to phase lock. This can be understood by looking at Eq. (42) and noting that phase locking is achieved when the $\alpha_{1}^{2} / \alpha_{2}$ part of the first term dominates over $2 \alpha_{2}$. In order for this to occur, $\alpha_{1}$ must grow from its initial small



FIG. 2. The walls of the confinement region are divided into rings of different lengths. The axial confining potentials are applied to the two $2.5 \mathrm{~cm}$ rings (numbers 1 and 9) at the ends of the plasma. The two $10 \mathrm{~cm}$ rings (numbers 3 and 7) are used as the detectors for the modes discussed in this paper.

state. The timescale for $\alpha_{1}$ to grow can be inferred from Eq. (40); Eq. (40) divided by $\alpha_{1}$ gives the timescale for changes in $\alpha_{1}$. We can see that the first part is proportional to $\alpha_{2}$, leading to longer times to phase lock for smaller initial $\alpha_{2}$. This is confirmed by numerical integration of the equations.

Note also that the damping is essential for the phase locking of this system. As shown by numerical experimentation with the model above, without any damping the modes just convert back and forth between each other. Also, if $\gamma_{e}$ $<0$ then phase locking never occurs at all.

It makes very little difference to the final state whether the plasma starts with a large $j=2$ mode or a large $j=1$ mode, since the plasma typically converts back and forth between these two modes more than once before the final state is achieved (see Fig. 1). Figure 1 starts with a large $j$ $=2$ mode, but it converts to mostly $j=1$ at $12 \mu \mathrm{s}$. Since the equations are first order in time, the system has no memory and the evolution would be the same after that time whether we just started with that initial condition or continued with the original calculation. The only significant difference between starting with large $j=2$ or $j=1$ is that starting with a large $j=1$ mode does not require the presence of any $j=2$ mode to create more of a $j=2$ mode, whereas starting with a large $j=2$ mode does require the presence of a small amount of the $j=1$ mode to start with to create more of a $j=1$ mode. Note the differences in Eqs. (36) and (37).

\section{EXPERIMENT}

Our experiment to observe this effect is a non-neutral (pure electron) plasma confined in a Malmberg-Penning $\operatorname{trap}^{4,5}$ operated in a fill-manipulate-dump cycle. The radial confinement is provided by an axial magnetic field and the axial confinement is provided by electric fields at the ends of the confinement region. See Fig. 2. The confinement voltages are applied to the rings labeled 1 and 9 in the figure. The electron plasma is created by thermionic emission from a spiral filament, confined and observed for several milliseconds and then dumped from the other end. The waves of interest are electrostatic TG waves created by oscillating the potential on the confining rings of the plasma. While such waves in a finite length plasma do not have exactly the spatial dependence shown in the theory earlier, that dependence is a good approximation as long as the plasma is long compared to its radius. ${ }^{1,2}$ Our experiment satisfies that criterion, the plasma being $60 \mathrm{~cm}$ long and about $2 \mathrm{~cm}$ in radius. TG waves have the characteristic that the dispersion relation is 


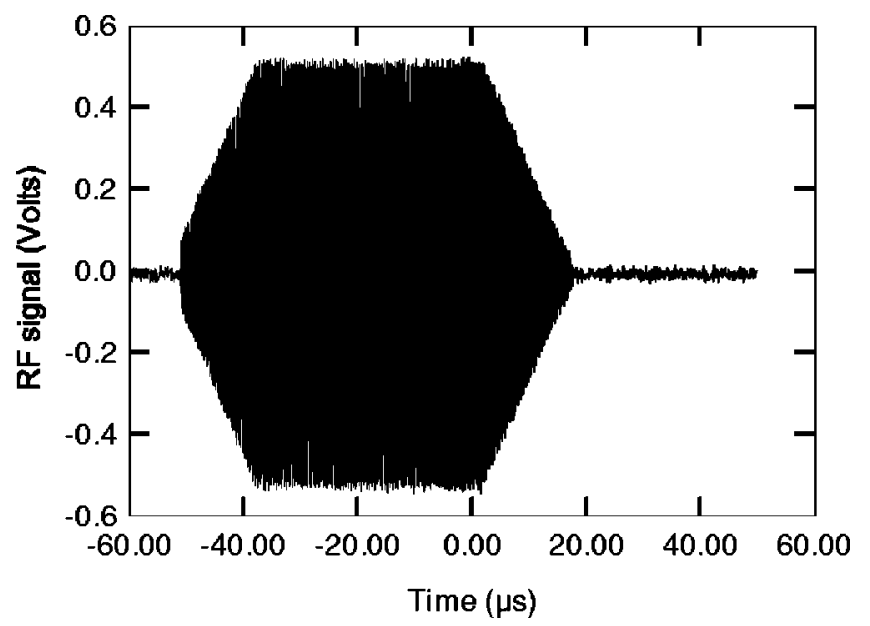

FIG. 3. The oscillating drive voltage applied to the end rings of the plasma as a function of time. The drive is turned on and off over a $15 \mu$ s period and is on for about $40 \mu \mathrm{s}$.

approximately linear at low $j$ and drops below the linear relationship as $j$ increases. We used a drift kinetic code, ${ }^{6}$ DRIFTK, to calculate the linear, uncoupled frequencies for our plasma profiles. There was less than a $1 \%$ frequency mismatch between the quantity $2 \omega_{2}-\omega_{1}$ and $\omega_{1}$, but about an $8 \%$ mismatch between $\omega_{2}+\omega_{1}$ and $\omega_{3}$. The frequency mismatch between $2 \omega_{2}$ and $\omega_{4}$ is about $20 \%$. The percentages are measured relative to $\omega_{1}$. This would imply that the amplitude of the $j=4$ mode should alternately grow and damp over a period of a few cycles, leading to no net effect. Experimentally, it is difficult to measure the unshifted frequency of the $j=2$ mode because the $j=1$ mode always couples to it and causes its frequency to lock to twice that of the $j=1$ mode even at a fairly low amplitude.

In this experiment we excited the $j=2$ mode by oscillating the confining potentials on both ends of the plasma near the mode frequency, $4.38 \mathrm{MHz}$, in our case. The driving signal is shown in Fig. 3. The driving signal was ramped up from zero to its maximum value in about $15 \mu \mathrm{s}$, stayed at its maximum value for about $40 \mu \mathrm{s}$ and then was ramped back to zero in about $15 \mu \mathrm{s}$ more. The drive was fully turned off by $18 \mu \mathrm{s}$. We took data with various values for the maximum amplitude of the drive. We have taken data where we excited the $j=1$ mode to start with, but they showed no significant differences from the data shown here.

We observed the oscillations in the plasma by looking at the surface charge induced by the changing plasma density in sections of the wall of the confinement region. The signal is approximately equal to the integral of the plasma charge density over the length of the wall section divided by the capacitance of the external circuit. The signals of the two modes can be separated by making use of the different symmetries of the modes. We recorded the charge oscillations in two 10 $\mathrm{cm}$ long sections of the wall that were symmetrically placed relative to the middle of the plasma (rings 3 and 7 in Fig. 2, located at \pm 15 to $\pm 25 \mathrm{~cm}$ ). When the two signals are added, the odd parity $j=1$ mode cancels out and we are left with the even parity $j=2$ mode. If the two signals are subtracted, we end up with only the $j=1$ mode. Higher-order even or odd modes can also show up in these signals. These particular wall sections have very little sensitivity to the $j=3$ mode because that mode has a node near $z=20 \mathrm{~cm}$. There was no evidence for the $j=4$ mode in our data.

In order to compare our data with the theory, these raw voltages need to be converted into $\alpha$ values. In the theory $\alpha_{j}=n_{j} / n_{0}$ where $n_{j}$ is the amplitude of the perturbed density of the $j$ th mode. The sensitivity of a given ring to a particular mode can be approximated by integrating the waveform of the perturbed charge over the length of the ring. For example, the charge induced on a ring located between $z_{b}$ and $z_{e}$ with a $j=1$ mode would be

$$
Q_{1}=\int_{z_{b}}^{z_{e}} n_{1} \sin \left(k_{1} z\right) d z=n_{1} S_{1},
$$

where $n_{1}$ is the charge/unit length of the mode (the perturbed density integrated over the cross-sectional area) and $Q_{1}$ is the charge induced on the ring due to mode 1 . This implies that the relationship between $V_{1}$, the voltage induced on the ring and the mode amplitude, is

$$
V_{1}=Q_{1} / C=n_{1} S_{1} / C,
$$

where $C$ is the capacitance of the ring and cables to ground. In order to find $\alpha$ we also need to know $n_{0}$. This can be found from the signal on the ring when the plasma is dumped. Using the same reasoning as above, the relationship for $V_{0}$ is found to be

$$
V_{0}=Q_{0} / C=n_{0} L_{r} / C,
$$

where $L_{r}$ is the length of the ring and $V_{0}$ is the size of the voltage spike when the plasma is dumped. So

$$
\alpha_{1}=\frac{n_{1}}{n_{0}}=\frac{V_{1} / S_{1}}{V_{0} / L_{r}}=V_{1} \frac{L_{r}}{V_{0} S_{1}} .
$$

An equivalent expression for $\alpha_{2}$ can also be written. The scale factors $S_{1}$ and $S_{2}$ need to be multiplied by a factor of 2 from the expression given above to take into account the fact that two rings are added or subtracted.

To compare the data with the model, we need to determine the amplitude, frequency and phase of the oscillations as a function of time. We did this by taking 3 microsecond pieces of the data and doing a nonlinear least-squares fit of the data to a sinusoidal function. A fast Fourier transform (FFT) is not adequate in this situation because it does not have enough frequency resolution and makes the implicit assumption that the signal is periodic with a three microsecond period. The least-squares method gives much higher frequency resolution (without the periodicity assumption) at the expense of losing any information about other frequencies present in the data.

We took data at five different driving amplitudes, $0.3 \mathrm{~V}$, $0.5 \mathrm{~V}, 1.0 \mathrm{~V}, 2.0 \mathrm{~V}$ and $3.0 \mathrm{~V}$. The amount of plasma response depended on two factors: the size of the drive and how closely the $j=2$ mode frequency matched the driving frequency of $4.38 \mathrm{MHz}$. Occasionally a shot with a lower drive would have a larger response than a shot with a larger drive because its natural frequency matched the drive better because of shot-to-shot variability. All shots with 2 and $3 \mathrm{~V}$ 


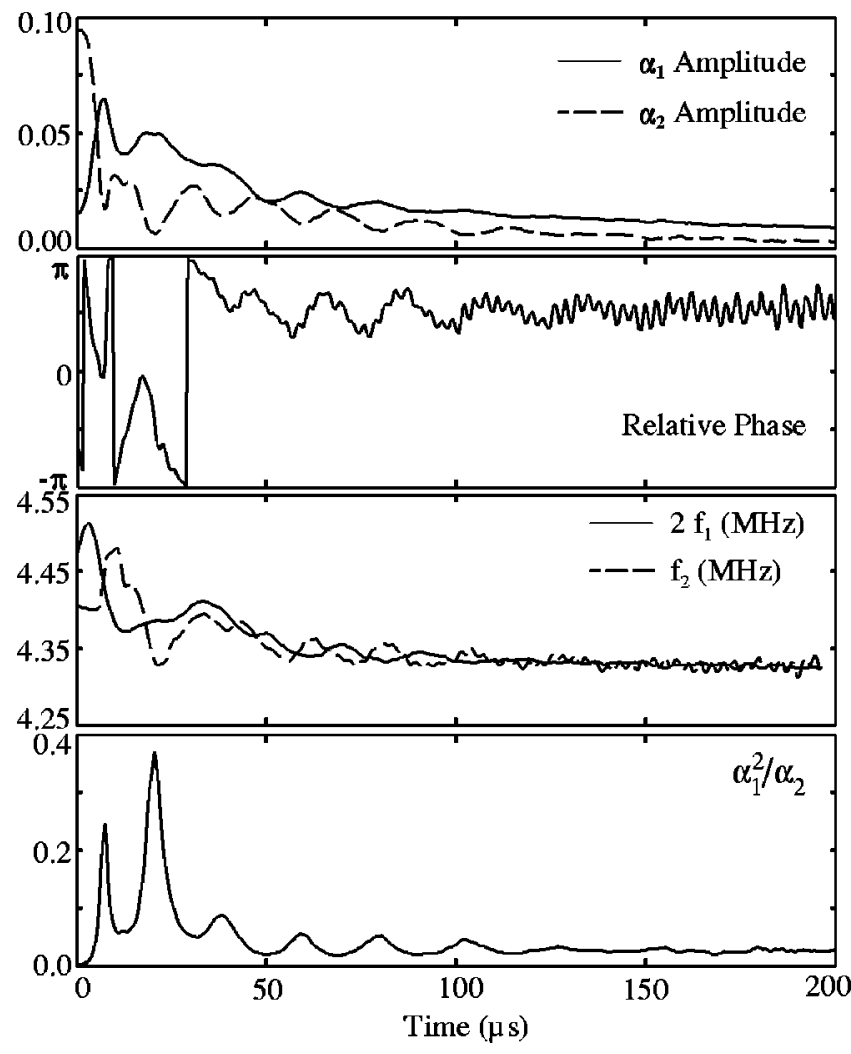

FIG. 4. Results of an analysis of a large amplitude wave. The top curve shows the normalized amplitudes of the two modes. The second plot shows the relative phase, the third shows $2 f_{1}$ and $f_{2}$ and the fourth shows the ratio $\alpha_{1}^{2} / \alpha_{2}$. This shot phase locked quickly.

drive were found to have solitons ${ }^{7,8}$ in them, which would substantially complicate the interpretation, and so they were excluded from further analysis. All shots with $\alpha_{1}<0.02$ on the first peak after the drive turned off did not phase lock within the time period of observation, although they appeared to be approaching a lock near the end of the data. Our data set included 8 shots with a significant plasma response. Five of them produced a final phase-locked state and three of them were too small to lock in the observation time.

Figures 4 and 5 show the results of applying this procedure to two sets of data with different drives. Figure 4 refers to a shot with a $1 \mathrm{~V}$ drive applied to the confining rings and represents the largest response obtained without solitons. Figure 5 refers to a shot with a $0.3 \mathrm{~V}$ drive and represents the smallest response that phase locked. The top plot of each data set shows the two mode amplitudes, with $\alpha_{2}$ being the dashed line and $\alpha_{1}$ being the solid line. The relative phase $\Phi=2 \phi_{1}-\phi_{2}$ is shown in the second plot. For both cases it locks near a value of $\pi / 2$ where $\tan (\Phi) \approx \infty$. This implies that $\gamma_{e} \ll 2 \delta \omega$. The third plot shows both $2 f_{1}$ and $f_{2}$ measured in MHz. Note that the presence of a $\dot{\phi}_{1}$ and $\dot{\phi}_{2}$ can be interpreted as a frequency shift. When the phase locks, these two frequencies must be equal. The bottom plot in each case is $\alpha_{1}^{2} / \alpha_{2}$ as a function of time. This ratio clearly approaches a constant value as the phase approaches a constant. The data in Fig. 5 start at $27 \mu$ s because the amplitude of the $j=2$ mode was too small before that time to make the data meaningful; it was smaller than the noise in the data.

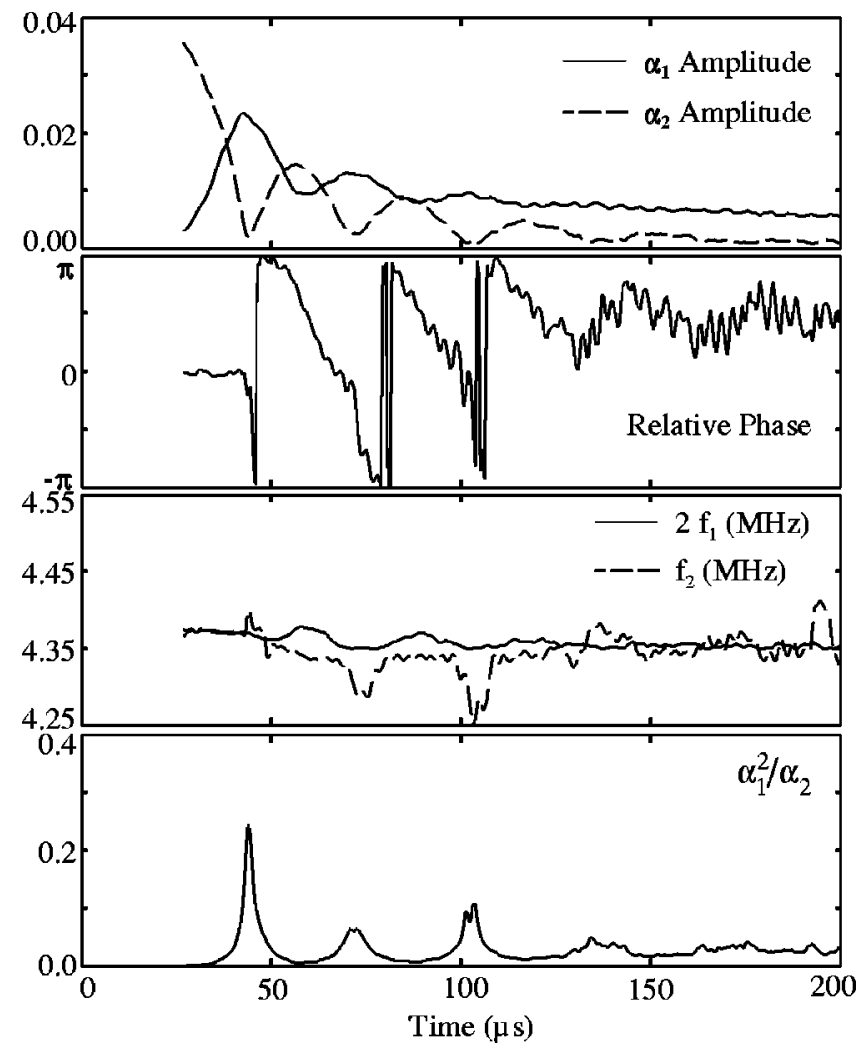

FIG. 5. Results of an analysis of a smaller amplitude wave. The plots are the same as in the previous figure. This shot took longer to phase lock.

It is clear in these data sets that in spite of their substantially different initial conditions, the final state is very similar. The higher amplitude case actually locks sooner and at a higher amplitude than the initially lower amplitude case. This is not surprising, since the mode conversion process occurs faster for a higher amplitude wave. This means that in the higher amplitude case the $j=2$ mode starts to convert to the $j=1$ mode well before the drive is turned off and both modes are locked to the drive. This can be seen in the fact that the frequencies of both modes are already equal before $t=0$ and never diverge very much. In the low amplitude case the $j=1$ mode does not start to grow until the drive is turned off. They are initially locked together with a relative phase of zero, since the $j=2$ mode is the source of the $j=1$ mode and the largest growth will occur with $\Phi=0$. When the amplitude of the $j=2$ mode drops to near zero, they decouple for a while and the frequencies diverge, only to converge again later. Note that it is the $j=2$ mode that has the much more variable frequency. We have data sets with smaller initial $\alpha_{2}$, but those cases take longer to lock than the length of the data record; they are showing signs of approaching the final state, such as the two frequencies converging, but have not yet achieved it by the end of the data.

The asymptotic values of $\Phi$ and $\alpha_{1}^{2} / \alpha_{2}$ can be estimated by averaging their values over the portion of the trace where they are roughly constant. When we do so on all the phaselocked shots in our data set we get values of $\alpha_{1}^{2} / \alpha_{2}=0.026$ and $\Phi=0.53 \pi$ with an uncertainty of about \pm 1 in the last digit of both values. If we define $R=\alpha_{1}^{2} / \alpha_{2}$ and $T$ $=\tan (\Phi)$, we can rearrange Eqs. (47) and (53) into 


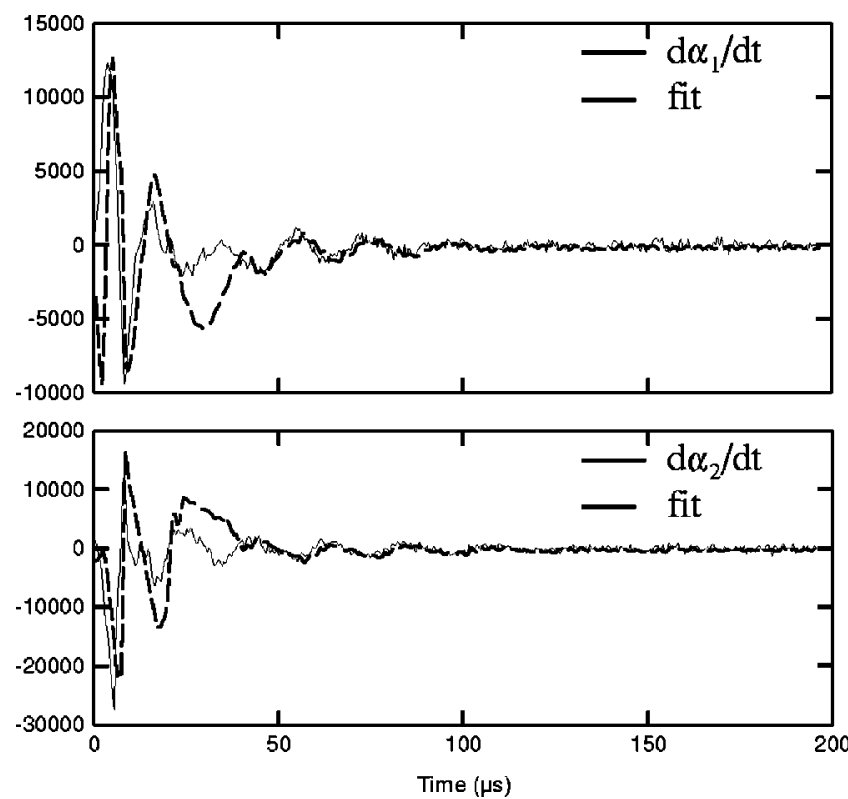

FIG. 6. Fits of $\dot{\alpha}_{1}$ and $\dot{\alpha}_{2}$ to the theoretical model for the large amplitude case above. The fits work well except at times when $j=3$ could reasonably be expected to be driven by the modes present.

$$
\begin{aligned}
& \frac{\delta \omega}{\omega_{1}}=\frac{3}{8} \frac{R}{\sqrt{1+1 / T^{2}}}, \\
& \frac{\gamma_{e}}{\omega_{1}}=\frac{3}{4} \frac{R}{\sqrt{1+T^{2}}} .
\end{aligned}
$$

Putting the above values for $R$ and $T=\tan (\Phi)=-15.9$ into these equations yields $\delta \omega / \omega_{1}=0.0098$ and $\gamma_{e} / \omega_{1}=1$ $\times 10^{-3}$. Because of the steep slope of $\tan (\Phi)$ near $\pi / 2$ and the dependencies on $T$ in the two equations, the uncertainty in $\gamma_{e} / \omega_{1}$ is about a factor of 2 , whereas the uncertainty in $\delta \omega / \omega_{1}$ is about \pm 3 in the last digit. This value of $\delta \omega / \omega_{1}$ is consistent with the theoretical value calculated from DRIFTK mentioned earlier. It is not possible to measure $\delta \omega$ directly from the data because by the time the modes are of measurable amplitude they are already interacting with the drive and each other.

In order to compare the data with the model in more detail, we refer to Eqs. (36) and (37). Taking the time derivative of our $\alpha_{1}$ and $\alpha_{2}$ curves, the data can be fit to these two equations using $\gamma_{1}$ and $\gamma_{2}$ as free parameters. Figures 6 and 7 show $\dot{\alpha}_{1}$ and $\dot{\alpha}_{2}$ and the fits. Figure 6 is the large amplitude case mentioned previously and Fig. 7 is the small amplitude case. The parameters were derived by fitting only the data in the time period from $40 \mu \mathrm{s}$ to $200 \mu \mathrm{s}$, but the fit is plotted using those parameters over the whole time period from 0 to $200 \mu \mathrm{s}$. The fit matches well for the entire plot in Fig. 7, but in Fig. 6 there are periods before $40 \mu$ s where the fit does not work well. The drive is not fully turned off until about $18 \mu \mathrm{s}$, so the model cannot be expected to work well for that time period. The model also assumes that no $j=3$ mode is present in the system to couple to the $\omega_{1}+\omega_{2}$ term. The deviation from the model during the time period from $18-40 \mu \mathrm{s}$ is possibly explained by coupling to the $j=3$

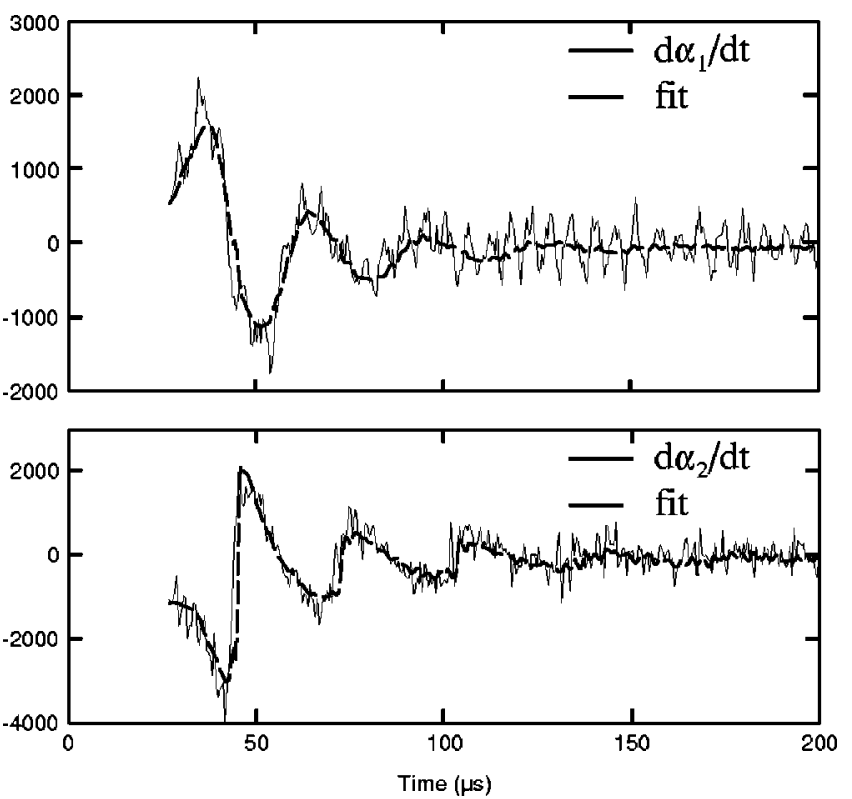

FIG. 7. Fits of $\dot{\alpha}_{1}$ and $\dot{\alpha}_{2}$ to the theoretical model for the small amplitude case above. The fits work well throughout the observation period.

mode. Note that $\alpha_{2}$ is not growing as fast as predicted by the model and that $\alpha_{1}$ is not shrinking as fast as predicted during that time period. This might possibly represent a transfer of energy from $\alpha_{2}$ to $\alpha_{1}$ through the intermediary of the $j=3$ mode. It is significant that this deviation occurs at a time when both modes are of relatively large amplitude.

The fits produced slightly different values of $\gamma$ from the two shots shown here. For Fig. 6 we get $\gamma_{1}=1.1 \times 10^{4} \mathrm{~s}^{-1}$ and $\gamma_{2}=5.4 \times 10^{4} \mathrm{~s}^{-1}$, giving $\gamma_{e} / \omega_{1}=2.3 \times 10^{-3}$. For Fig. 7 we get $\gamma_{1}=1.8 \times 10^{4}$ and $\gamma_{2}=6.0 \times 10^{4}$. This implies $\gamma_{e} / \omega_{1}=1.8 \times 10^{-3}$. If we average the values from our whole data set of shots that phase locked we get values of $\gamma_{1}$ $=1.2 \pm 0.3 \times 10^{4}$ and $\gamma_{2}=5.6 \pm 0.7 \times 10^{4}$, yielding $\gamma_{e} / \omega_{1}$ $=2.3 \pm 1.0 \times 10^{-3}$. This is consistent with the results of the asymptotic state analysis given above. Note that these values of $\gamma_{1}$ and $\gamma_{2}$ are consistent with the condition that $\gamma_{2}$ $>2 \gamma_{1}$.

Another interesting question is what happens to the energy that was initially in the $j=2$ mode. The small-signal energy density of the mode is proportional to

$$
E_{r}^{2}+E_{z}^{2}\left(1+\left(\frac{\omega_{p}}{\omega}\right)^{2}\right)
$$

where the $\omega_{p} / \omega$ part represents the kinetic energy of the mode and the rest is the potential energy. The kinetic energy only links to $E_{z}$ because this mode only has motion in the $z$-direction.

The connection between the $\alpha_{j}$ 's and the mode energy can be obtained by starting with the mode density. If the modes are assumed to have the same radial dependence, call it $R\left(k_{r} r\right), n_{j}$ is just

$$
n_{j}=\overline{n_{j}} R\left(k_{r} r\right)\left\{\begin{array}{ll}
\sin \left(k_{z} z\right) & \text { odd } j \text { modes } \\
\cos \left(k_{z} z\right) & \text { even } j \text { modes }
\end{array}\right\} \text {, }
$$


where $\overline{n_{j}}$ is the maximum amplitude of the $j$ th mode and $k_{z}=2 \pi j / L$. From our definition of $\alpha_{j}$, we know that $\alpha_{j}$ $=\overline{n_{j}} / n_{0}$. Equation (15) provides the link between the potential and the density, so that $\phi_{j}$ is

$$
\phi_{j}=\frac{\alpha_{j} n_{0} R\left(k_{r} r\right)}{\left(k_{r}^{2}+k_{z}^{2}\right)}\left\{\begin{array}{ll}
\sin \left(k_{z} z\right) & \text { odd } j \text { modes } \\
\cos \left(k_{z} z\right) & \text { even } j \text { modes }
\end{array}\right\} .
$$

Given the mode energy density given in Eq. (60) above, the total mode energy will be

$$
W_{j} \propto \int_{0}^{a} 2 \pi r d r \int_{-L / 2}^{L / 2} d z\left[E_{r}^{2}+E_{z}^{2}\left(1+\frac{\omega_{p}^{2}}{\omega^{2}}\right)\right],
$$

where $a$ is the wall radius. Writing this in terms of the potential gives

$$
\begin{aligned}
W_{j} & \propto \int_{0}^{a} 2 \pi r d r \int_{-L / 2}^{L / 2} d z\left[\left(\frac{\alpha_{j} k_{r} R^{\prime}\left(k_{r} r\right)}{\left(k_{r}^{2}+k_{z}^{2}\right)}\right)^{2}\right. \\
& \times\left\{\begin{array}{ll}
\sin ^{2}\left(k_{z} z\right) & \text { odd } j \text { modes } \\
\cos ^{2}\left(k_{z} z\right) & \text { even } j \text { modes }
\end{array}\right\} \\
& +\left(\frac{\alpha_{j} k R\left(k_{r} r\right)}{\left(k_{r}^{2}+k_{z}^{2}\right)}\right)^{2}\left(1+\frac{\omega_{p}^{2}}{\omega^{2}}\right) \\
& \left.\times\left\{\begin{array}{lll}
\cos ^{2}\left(k_{z} z\right) & \text { odd } j \text { modes } \\
\sin ^{2}\left(k_{z} z\right) & \text { even } j \text { modes }
\end{array}\right\}\right],
\end{aligned}
$$

where all common factors that are not mode dependent have been factored out, since this is a proportionality.

When the $z$-dependence shown in Eq. (64) is integrated, it produces $L / 2$ for all modes, since the integral is over a whole number of half wavelengths. We note that $\omega_{p} / \omega$ is a large number, of order 10, and so we can ignore the 1 relative to $\omega_{p}^{2} / \omega^{2}$. Noting that $k^{2}=\omega^{2} / v_{p}^{2}$, where $v_{p}$ is the approximately mode-independent phase velocity of the wave, this expression simplifies to

$$
W_{j} \propto \frac{\alpha_{j}^{2}}{\left(k_{r}^{2}+k_{z}^{2}\right)^{2}} \int_{0}^{a} r d r\left[\left(k_{r} R^{\prime}\left(k_{r} r\right)\right)^{2}+\left(\frac{R\left(k_{r} r\right) \omega_{p}}{v_{p}}\right)^{2}\right] .
$$

The only remaining mode dependence in this expression is the $\left(k_{r}^{2}+k_{z}^{2}\right)^{2}$ in the denominator. $k_{r}$ is of order $1 / a$ and $k_{z}$ is of order $1 / \lambda$, where $\lambda$ is the wavelength of the wave in the $z$ direction. For our experimental configuration $k_{r}$ is much larger than $k_{z}$ unless the mode number is very high, so the $k_{z}^{2}$ term can be removed, making the initial constant mode independent. This leads us to the conclusion that

$$
W_{j}=C \alpha_{j}^{2},
$$

where the constant $C$ includes all the factors that are constant between the two modes.

Without determining the constant $C$, it is possible to study the transfer of energy between the two modes. Figures 8 and 9 show this for the same two cases shown above. Figure 8 is the high amplitude case. In both plots the three curves are the energy in the $j=2$ mode, the energy in the $j$ $=1$ mode and the total energy. At late times (after about 50

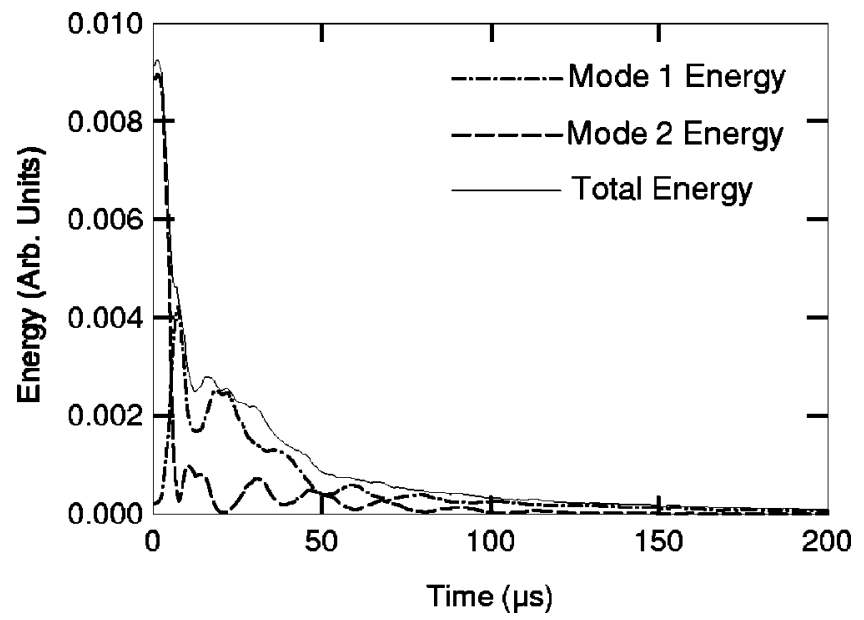

FIG. 8. The energy of the modes in the system for the large amplitude case. The dotted line is the energy in the $j=2$ mode, the dashed line is the energy in the $j=1$ mode and the solid line is the total energy.

$\mu \mathrm{s})$ both plots show the energy decaying at the rate appropriate for the mode which is dominant at that time. Before 50 $\mu \mathrm{s}$ there is an interesting difference. While Fig. 9 shows exponential decay at the dominant mode's rate, the high amplitude case (Fig. 8) shows more complicated behavior. The total energy decays too rapidly between 0 and $10 \mu \mathrm{s}$ and is too high between 20 and $40 \mu \mathrm{s}$. This is perhaps an indication of energy initially being stored in the $j=3$ mode and partially given back later. The high frequency oscillation seen in the total energy before $50 \mu \mathrm{s}$ is at about the expected beat frequency between $\omega_{2}+\omega_{1}$ and $\omega_{3}$.

\section{CONCLUSIONS}

In conclusion, we have used the cold fluid equations and the averaging method to derive a set of equations to describe the nonlinear coupling of the fundamental and second harmonic $(j=1$ and $j=2)$ Trivelpiece-Gould modes in a nonneutral plasma. These equations match the data well, as long as the assumptions inherent in the derivation are not violated.

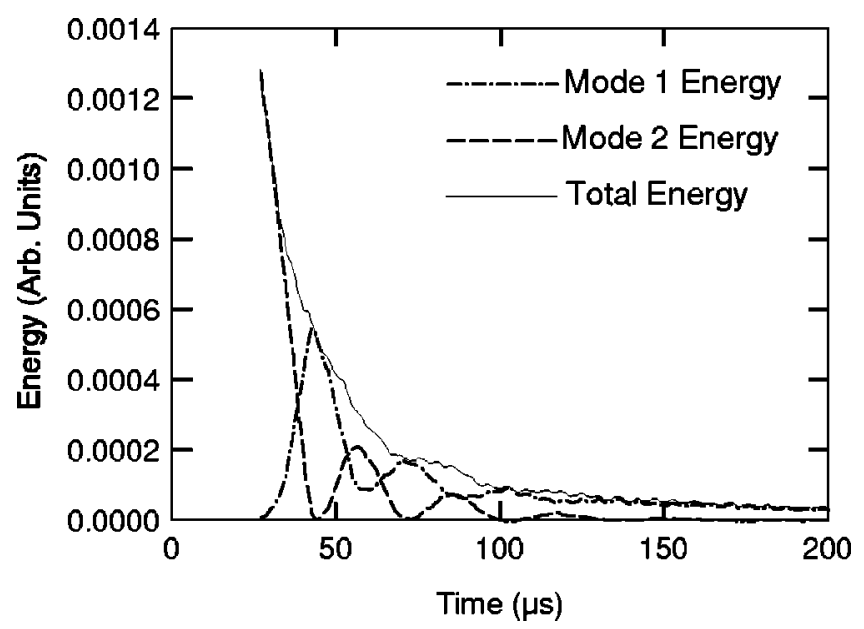

FIG. 9. The energy of the modes in the system for the small amplitude case. The dotted line is the energy in the $j=2$ mode, the dashed line is the energy in the $j=1$ mode and the solid line is the total energy. 
In particular, there is some evidence that the $j=3$ mode (third harmonic) can be present when the amplitude of both modes is particularly large and affect the coupling of these modes. If the amplitude of the waves is too large, then the model does not work well either, probably because of the presence of solitons in the plasma.

${ }^{1}$ S. A. Prasad and T. M. O’Neil, Phys. Fluids 26, 665 (1983).

${ }^{2}$ J. K. Jennings, R. L. Spencer, and K. C. Hansen, Phys. Plasmas 2, 2630 (1995).
${ }^{3}$ For a description of this method see, for example, Appendix C of R. Baierlein, Newtonian Dynamics (McGraw-Hill, New York, 1983), p. 317.

${ }^{4}$ J. S. deGrassie and J. H. Malmberg, Phys. Rev. Lett. 39, 1077 (1972).

${ }^{5}$ G. W. Hart, Phys. Fluids B 3, 2987 (1991).

${ }^{6}$ G. W. Mason and R. L. Spencer, Phys. Plasmas 9, 3217 (2002).

${ }^{7}$ G. W. Hart, R. L. Spencer, and B. G. Peterson, in Non-neutral Plasma Physics III, edited by J. J. Bollinger, R. L. Spencer, and R. C. Davidson (American Institute of Physics, Melville, NY, 1999), pp. 182-191.

${ }^{8}$ G. W. Hart and B. G. Peterson, in Non-neutral Plasma Physics IV, edited by F. Anderegg, L. Schweikhard, and C. F. Driscoll (American Institute of Physics, Melville, NY, 2002), pp. 341-346. 